\title{
Computing images of polynomial maps
}

\section{Corey Harris $^{1}$ - Mateusz Michałek ${ }^{1,2,3}$ (D) Emre Can Sertöz ${ }^{1}$}

Received: 23 October 2018 / Accepted: 11 July 2019 /

Published online: 8 August 2019

(C) The Author(s) 2019

\section{Abstract}

The image of a polynomial map is a constructible set. While computing its closure is standard in computer algebra systems, a procedure for computing the constructible set itself is not. We provide a new algorithm, based on algebro-geometric techniques, addressing this problem. We also apply these methods to answer a question of W. Hackbusch on the non-closedness of site-independent cyclic matrix product states for infinitely many parameters.

Keywords Polynomial maps · Constructible set · Matrix product states

Mathematics Subject Classification (2010) Primary 14Q15 · Secondary 68U05 · 15A69

\section{Introduction}

Determining the image of a polynomial map is of fundamental importance in numerous disciplines of mathematics. In particular, this problem comes up in dealing with parametrizations of (unirational) varieties, a situation which arises frequently in theory and in application, for instance in low-rank tensor approximation.

Given a projective variety $X \subset \mathbb{P}_{\mathbb{C}}^{n}$, we compute the image of a polynomial map $f: X \rightarrow \mathbb{P}_{\mathbb{C}}^{m}$. This setting easily extends to rational maps from affine varieties to affine spaces (see Section 2.1).

Our primary goal is to develop an algorithm to compute this image. We have two design principles regarding the output: first, it should give immediate insight to a human, and second, a computer using our output should be able to determine instantly

Communicated by: Ivan Oseledets

Mateusz Michałek

michalek@mis.mpg.de

1 Max Planck Institute for Mathematics in the Sciences, Leipzig, Germany

2 Institute of Mathematics of the Polish Academy of Sciences, Warsaw, Poland

3 Aalto University, Espoo, Finland 
if a point in the codomain belongs to the image. Let us emphasize here that the output we produce will make it clear at first sight whether or not the image is closed.

We begin with a simple example. Consider the Cremona transformation $f$ : $\mathbb{P}^{2} \rightarrow \mathbb{P}^{2}$ defined by $\left[x_{0}, x_{1}, x_{1}\right] \mapsto\left[x_{1} x_{2}, x_{0} x_{2}, x_{0} x_{1}\right]$. For more complicated examples, see Section 5.

Let us write the image of $f$ as a constructible set $V_{0} \backslash\left(V_{1} \backslash V_{2}\right)$ where $V_{0}=\mathbb{P}^{2}$, $V_{1}=Z\left(y_{0} y_{1} y_{2}\right)$, and $V_{2}=Z\left(y_{0} y_{1}, y_{0} y_{2}, y_{1} y_{2}\right)$. Here we represent closed algebraic sets as the zeros of an ideal, written $Z(I)$. It is more convenient however to decompose the $V_{i}$ 's into their irreducible components and store the containment relations in the form of a graph.

Then $V_{1}=L_{0} \cup L_{1} \cup L_{2}$ and $V_{2}=p_{0} \cup p_{1} \cup p_{2}$ where the $L_{i}$ 's (resp. $p_{i}$ 's) are the three lines (resp. points) in $\mathbb{P}^{2}$ defined by the vanishing of coordinates. The image of the Cremona transformation can now be presented as a graph as in Fig. 1a.

In our implementation, the image is represented in the form of a tree. For the Cremona transformation, it is depicted in Fig. 1b; meanwhile, the output of our implementation is presented in Fig. 2.

Standard methods exist for determining the closure of the image. They rely on Gröbner basis computations and are implemented in any general mathematical software, cf. \$3.3 [5]. As far as we are aware, however, the only software which computes the image of a polynomial map is PolynomialMapImage in the Maple ${ }^{\mathrm{TM}}$ module RegularChains [3, 19]. This program uses triangular decompositions-a technique well-developed in algorithmics [31].

Our algorithm relies on a central technique in algebraic geometry: resolving a rational map through blowups. Our Macaulay2 [8] implementation of the algorithm

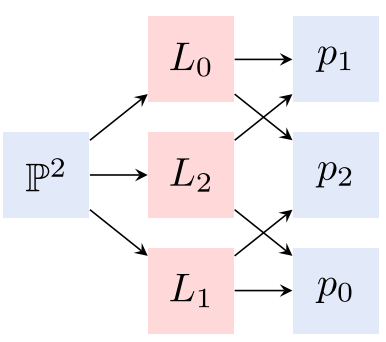

(a) Constructible graph

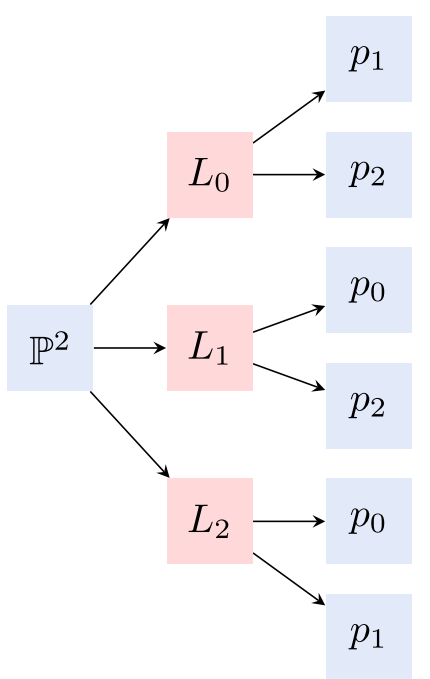

(b) Constructible tree

Fig. 1 Cremona transformation. a Constructible graph. b Constructible tree 


$$
\begin{aligned}
& \text { (2) ideal() } \\
& \text { - (1) } \mid====i d e a l \text { y2 } \\
& + \text { (0) }|\quad|====i d e a l(y 2, y 0) \\
& \text { + (0) | |====ideal (y2,y1) } \\
& \text { - (1) } \mid====i d e a l \text { y1 } \\
& + \text { (0) }|\quad|====i d e a l(y 1, y 0) \\
& \text { + (0) | | |====ideal (y2, y1) } \\
& \text { - (1) } \mid====i d e a l \text { y0 } \\
& + \text { (0) }|\quad|====i d e a l(y 2, y 0) \\
& + \text { (0) }|\quad|====i d e a l(y 1, y 0)
\end{aligned}
$$

Fig. 2 The output of our Total Image for the Cremona transformation

is called Total Image. ${ }^{1}$ It is typically much faster than PolynomialMapImage in our tests (see Section 3.3 for a detailed comparison).

We also demonstrate how one can make theoretical use of the idea behind this algorithm to prove that an image is not closed without computing the entire image. In the process, we prove that the set of tensors that admit site-independent matrix product state (IMPS) representations with fixed rank is not closed (Theorem 4.5). This answers a question posed by W. Hackbusch. A cousin problem of deciding whether the set of tensors that admits a matrix product state (MPS) representation forms a closed set, posed by L. Grasedyck, was settled in [18].

We will now describe three domains of application in which the determination of the image of a map plays a crucial role.

\subsection{Physics}

Tensors play a prominent role in physics, for instance in the representation of quantum states. An issue is that relevant tensors often appear in spaces of huge dimension, making them practically impossible to work with directly.

A way around this problem is to find compact representations of a tensor, such as low-rank presentations (also known as the canonical polyadic decomposition) or tensor networks [11]. In practice, one often gives an algebraic parametrization of a family of well-behaving tensors, such as those admitting compact representations. It is of great concern from the point of view of numerical mathematics to decide whether the image of such a parametrization map is closed.

\footnotetext{
${ }^{1}$ Available at https://github.com/coreysharris/TotalImage
} 
In other words, one wishes to know in advance whether a sequence of wellbehaving tensors $T_{n}$, approximating an arbitrary tensor $T$, will converge to a good approximation $T_{\infty}$ within the set of well-behaving tensors. For example, for a specified $r$ and a real tensor $T$, there may be no best-possible real-rank $r$ approximation of $T$. In fact, this happens with positive probability in the choice of $T$ [7]. The complex case, where such phenomena do not take place, along with examples when best rank approximations do not exist, is discussed in [24].

\subsection{Statistics}

A statistical model is a parametric family of probability distributions. A large class of statistical models are parametrized by algebraic maps [6, 21, 26].

The primary question about a statistical model is if a given, i.e., observed, probability distribution fits the model. To attack this question, one wishes to describe the real image of the parametrization corresponding to the model within the space of all probability distributions.

In this paper, we only deal with the complex image of algebraic maps. However, the complex image, being larger, often gives a good first test for the fitness of a statistical model.

\subsection{Computational sciences}

Tensors represent multi-linear maps. Good representations of a tensor, for instance its rank decomposition, yield algorithms of lower complexity $[16,17]$.

A famous example demonstrating this relationship is matrix multiplication. The multiplication of two $n \times n$ matrices is a bilinear operation and thus is represented by a 3-dimensional tensor. The complexity of the optimal algorithm for multiplying matrices is known to be governed by the rank (or border rank) of the associated tensor (see $[16,17]$ ). (Let us point out that it is not known in general if the rank and the border rank of the matrix multiplication tensor coincide.)

Computing the tensor rank (as well as determining if the tensor rank equals the border rank) of a given tensor $T$ is equivalent to the problem of deciding whether $T$ belongs to the image of an algebraic map (or its closure).

We start by presenting the preliminaries in Section 2. In Section 3, we present our algorithm for computation of images. In Section 4, we answer the question of W. Hackbusch proving that IMPS tensors do not form a closed set in general. In Section 5, we present in detail two explicit examples inspired by statistics and physics. Some of the proofs and remarks are postponed to the Appendix.

\section{Preliminaries}

A map $f: \mathbb{C}^{n} \rightarrow \mathbb{C}^{m}$ defined by $x \mapsto\left(f_{1}(x), \ldots, f_{m}(x)\right)$ where the $f_{i}$ are polynomials in the coordinates of $x=\left(x_{1}, \ldots, x_{n}\right)$ is called a polynomial map. If the $f_{i}$ are given as the quotient of two polynomials, then $f$ is called a map of rational functions. 
Note that if the $f_{i}$ are not polynomials, the map $f$ is not well defined everywhere in the domain and we use the notation $f: \mathbb{C}^{n}-\rightarrow \mathbb{C}^{m}$ to allow for this possibility.

The goal of this paper is to compute the image of a map of rational functions. There is another case of interest however which turns out to generalize the one above while providing a more advantageous perspective.

Consider a map $f: \mathbb{P}^{n} \rightarrow \mathbb{P}^{m}$ defined by $[x] \mapsto\left[f_{0}(x), \ldots, f_{m}(x)\right]$ where the $f_{i}$ are rational functions. This makes sense only when the $f_{i}$ are homogeneous of the same degree.

When each $f_{i}$ is a polynomial, we may emphasize this fact by referring to $f$ as a polynomial map. Note that even when $f$ is a polynomial map, $f$ need not be well defined on the entire domain, and we will use the notation $\mathbb{P}^{n} \rightarrow \mathbb{P}^{m}$ to highlight this fact.

\subsection{Changing affine domain into projective domain}

Suppose we are given a map $f: \mathbb{C}^{n} \rightarrow \mathbb{C}^{m}$ defined by rational functions. We will now show that computing the image of $f$ can be reduced to computing the image of a polynomial map between the projective spaces $\mathbb{P}^{n} \rightarrow \rightarrow \mathbb{P}^{m}$. The standard way to do this would change the image; the trick below allows one to perform this extension without changing the image.

With $x_{0}, \ldots, x_{m}$ the homogeneous coordinates on $\mathbb{P}^{m}$, we identify $\mathbb{C}^{m}$ with the affine chart $\left\{x_{0}=1\right\}$ in $\mathbb{P}^{m}$ via the inclusion map $\iota: \mathbb{C}^{m} \rightarrow \mathbb{P}^{m}$ defined by $\left(x_{1}, \ldots, x_{m}\right) \mapsto\left[1, x_{1}, \ldots, x_{m}\right]$. The map $\iota \circ f: \mathbb{C}^{m} \hookrightarrow \mathbb{P}^{m}$ extends to $\tilde{f}: \mathbb{P}^{n} \rightarrow$ $\mathbb{P}^{m}$ as the map

$$
\left[x_{0}, \ldots, x_{n}\right] \mapsto\left[1, x_{0}^{-\operatorname{deg} f_{1}} f_{1}, \ldots, x_{0}^{-\operatorname{deg} f_{m}} f_{m}\right]
$$

defined by homogeneous rational functions of degree 0 . Observe that $\tilde{f}$ is not defined at the hyperplane at infinity $\left\{x_{0}=0\right\}$. Furthermore, on the affine chart $\left\{x_{0}=1\right\}$ of the domain-to be identified with $\mathbb{C}^{n}$ - the map $\tilde{f}$ is undefined precisely where $f$ is undefined. In particular, the maps $f$ and $\tilde{f}$ have the same image.

Further, we can convert any rational map $f: \mathbb{P}^{n} \rightarrow \mathbb{P}^{m}$, defined by rational functions $f_{i}=\frac{g_{i}}{h_{i}}$ of the same degree, to a polynomial map without changing the image. Set $H:=\prod_{i=0}^{m} h_{i}$ and define the polynomial map

$$
g=\left[H^{2} f_{0}, H^{2} f_{1}, \ldots, H^{2} f_{m}\right]
$$

The images of $f$ and $g$ coincide, although $g$ is a polynomial.

For these reasons, we may concentrate on computing the image of polynomial maps $\mathbb{P}^{n} \rightarrow-\mathbb{P}^{m}$ and their restrictions to varieties $X$ in $\mathbb{P}^{n}$ without loss of generality.

\subsection{Constructible sets}

Let $X$ be a topological space. A subset $Y \subset X$ is called a locally closed set if there exist open sets $U_{1}, U_{2} \subset X$ such that $Y=U_{1} \cap U_{2}^{c}$. A subset $Y \subset X$ is called 
a constructible set if $Y$ is the union of many finitely locally closed subsets. Given closed sets $V_{0}, \ldots, V_{k} \subset X$, we will denote the constructible set

$$
V_{0} \backslash\left(V_{1} \backslash\left(V_{2} \backslash\left(\ldots\left(V_{k-1} \backslash V_{k}\right) \ldots\right)\right)\right)
$$

by the alternating sum $V_{0}-V_{1}+\cdots+(-1)^{k} V_{k}$.

Definition 2.1 For a constructible set $C$, a representation $C=V_{0}-V_{1}+\cdots+$ $(-1)^{k} V_{k}$ will be called canonical if the following properties hold:

$$
\begin{aligned}
V_{2 s} & =\overline{V_{2 s-1} \cap C}, \\
V_{2 s+1} & =\overline{V_{2 s} \backslash C},
\end{aligned}
$$

for every $s \geq 0$, where we define $V_{-1}$ to be the ambient space of $C$.

Lemma 2.2 If $X$ is an algebraic variety and $C \subset X$ is a constructible set, then there exists a unique canonical representation of $C$.

Proof Uniqueness of the representation follows from the recursive definition provided above. As for existence, it is clear that if $V_{l}=\varnothing$ for some $l$, then $C=$ $V_{0}-V_{1}+\cdots+(-1)^{k} V_{l}$. Since $X$ is a Noetherian topological space, there is no strictly decreasing infinite sequence of closed sets in $X$. Therefore, it suffices to show that if $V_{2 s} \neq \emptyset$, then $V_{2 s+1}$ is strictly smaller than $V_{2 s}$. As we can replace $X$ with $V_{2 s}$ and $C$ with $C \cap V_{2 s}$, we only need to show $V_{1}$, the closure of $\bar{C} \backslash C$, is strictly smaller than $V_{0}=\bar{C}$, provided $V_{0} \neq \emptyset$. As $C$ is dense in its closure, the maximal dimension of the components of $V_{0}$ is strictly larger than the maximal dimension of the components of $V_{1}$.

\section{Image of a variety}

In this section, we describe our algorithm for computing the image of a polynomial map defined on a projective variety. Let us emphasize that we work over the complex numbers and point to references $[5,25]$ for the basic facts we will be using from algebraic geometry.

Our starting point is Chevalley's theorem on constructible sets.

Theorem 3.1 (Chevalley) Let $f: \mathbb{P}^{n} \rightarrow \mathbb{P}^{k}$ be a rational map and $V \subset \mathbb{P}^{n} a$ variety. If $B$ is the base locus of $f$, then $f(V \backslash B)$ is a constructible set.

In other words, the image can be described by a finite sequence of algebraic sets $\left(V_{0}, \ldots, V_{k}\right)$ such that:

- $\quad V_{0} \supsetneq V_{1} \supsetneq \cdots \supsetneq V_{k}$,

- $\operatorname{Im}(f)=V_{0}-V_{1}+V_{2}-\cdots+(-1)^{k} V_{k}$.

Here we note that the subtraction and addition operations on sets do not commute, and we are relying on our conventions in Section 2.2. 


\subsection{Presenting constructible sets as graphs.}

Throughout, graphs are simple and connected. A graph $\mathcal{G}$ with a distinguished vertex $\mathfrak{r}$ and no cycles is called a tree. The vertex $\mathfrak{r}$ is called the root. On each edge of $\mathcal{G}$, we can choose an orientation so that the edge points away from $\mathfrak{r}$. With this orientation, we will view our trees as being directed graphs.

Let $\mathcal{T}$ be a tree. If $\mathfrak{n} \rightarrow \mathfrak{n}^{\prime}$ is an edge of $\mathcal{T}$, then $\mathfrak{n}^{\prime}$ is called a child of $\mathfrak{n}$, and $\mathfrak{n}$ is called the parent of $\mathfrak{n}^{\prime}$. The vertices with no children are called leaves. The root is the only vertex with no parent. A tree has a natural grading, called depth, given by the path length from the root.

We can represent an irreducible constructible set $C$ as a tree with vertices labeled by varieties. The construction is inductive. If $C$ is closed, we represent it as a tree with a single vertex $\mathfrak{r}$ labeled by $C$. Otherwise, let $V_{i}$ be the irreducible components of $\bar{C} \backslash C$, and by induction, let $\mathcal{T}_{i}$ be the tree representation of the constructible set $V_{i}$. The tree $\mathcal{T}$ corresponding to $C$ is then constructed as follows: we label the root $\mathfrak{r}$ of $\mathcal{T}$ by the closure $\bar{C}$ and then attach the root of each $\mathcal{T}_{i}$ to $\mathfrak{r}$.

Definition 3.2 Any labeled tree obtained from a constructible set by the construction above will be called a constructible tree.

From the tree $\mathcal{T}$, we can recover the corresponding constructible set $C(\mathcal{T})$ inductively as follows:

$$
C(\mathcal{T})=V(\mathfrak{r}) \backslash \bigcup_{\mathfrak{n} \in \operatorname{children}(\mathfrak{r})} C\left(\mathcal{T}_{\mathfrak{n}}\right),
$$

where $\mathcal{T}_{\mathfrak{n}} \subset \mathcal{T}$ is the subtree with root $\mathfrak{n}$.

Lemma 3.3 Let $\mathcal{T}$ be a constructible tree with two vertices $\mathfrak{n}, \mathfrak{n}^{\prime} \in \mathcal{T}$. If $V(\mathfrak{n})=$ $V\left(\mathfrak{n}^{\prime}\right)$, then $\operatorname{depth}(\mathfrak{n}) \equiv \operatorname{depth}\left(\mathfrak{n}^{\prime}\right)(\bmod 2)$.

Proof The parity of depth determines whether or not the generic point of $V(\mathfrak{n})=$ $V\left(\mathfrak{n}^{\prime}\right)$ is contained in $C(\mathcal{T})$.

Therefore, to obtain a constructible graph from a constructible tree, we may identify vertices having the same label (Fig. 3).

Remark 3.4 For our main algorithm, it seems more natural to define and use constructible trees. However, constructible graphs are a more compact representation of a constructible set. An implementation utilizing the graph structure would likely yield savings in time and memory (cf. [9, 13]).

Remark 3.5 If $f: \mathbb{A}^{n} \rightarrow \mathbb{A}^{m}$ is given by monomials, the constructible graph representing $\operatorname{Im}(f)$ is a subposet of the face lattice of the Newton polytope of $f$ (see [10]). 


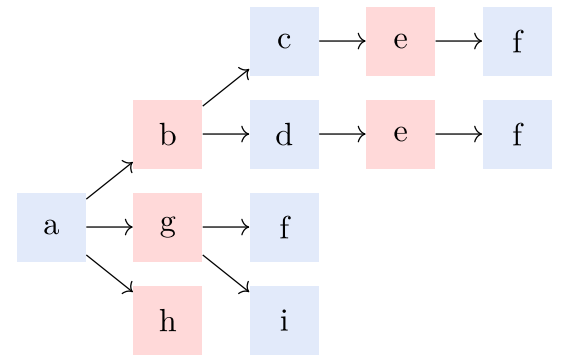

(a) Constructible tree

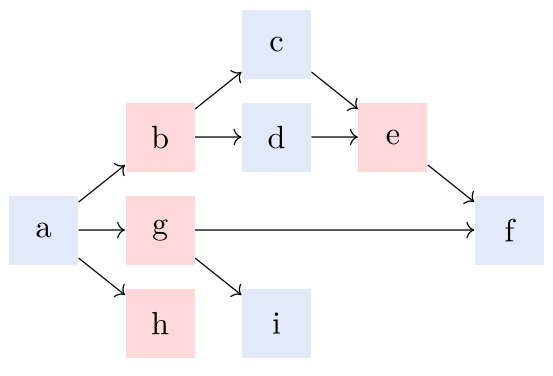

(b) Constructible graph

Fig. 3 A constructible tree and the resulting constructible graph. a Constructible tree. b Constructible graph

In general, if $f$ is a toric map, then $\operatorname{Im}(f)$ is a subposet of the face lattice of the polytope of characters defining $f$.

\subsection{An algorithm for computing images}

Let $X \subset \mathbb{P}^{n}$ be a variety and $f: X \rightarrow \mathbb{P}^{m}$ be a polynomial map $x \mapsto\left[f_{0}(x): \cdots\right.$ : $\left.f_{m}(x)\right]$ in the coordinates of $\mathbb{P}^{n}$.

Definition 3.6 The indeterminacy locus of $f$ is the subvariety of $X$ cut out by the ideal $\left(f_{0}, \ldots, f_{m}\right)+I_{X}$. This variety will be denoted by $\mathcal{B}_{f}$. The image of $f$, denoted $\operatorname{Im}(f)$, is the set $f\left(X \backslash \mathcal{B}_{f}\right)$. The image closure of $f$, denoted $\overline{\operatorname{Im}}(f)$, is the Zariski closure of the image of $f$.

Definition 3.7 An algebraic set $A \subsetneq \overline{\operatorname{Im}}(f)$ containing the difference $\overline{\operatorname{Im}}(f) \backslash$ $\operatorname{Im}(f)$ will be called a (image) frame of $f$, since it covers the boundary of the image.

We start by describing a subroutine Frame which computes an image frame of $f: X \rightarrow \mathbb{P}^{m}$.

The idea is to resolve the map $f$ by the blowup $\mathrm{Bl}_{f} X$ of $X$ along the indeterminacy locus $B$ and compute the image of the exceptional divisor.

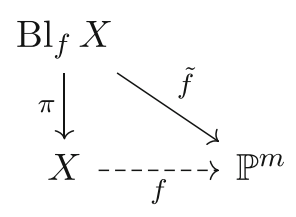

However, if $\operatorname{dim} X$ is strictly greater than the dimension of the image, the images of the exceptional divisors may dominate the image of $f$. To resolve this issue, we will cut down the dimension of $X$ by taking an appropriate linear section of $X$. 
Definition 3.8 Let $\Lambda \subset \mathbb{P}^{n}$ be a linear space and $\delta=\operatorname{dim} X-\operatorname{dim} X \cap \Lambda$. Then $X \cap \Lambda$ is a codimension $\delta$ linear section of $X$. If $\overline{\operatorname{Im}}(f)=\overline{\operatorname{Im}}\left(\left.f\right|_{X \cap \Lambda}\right)$, then the linear section $X \cap \Lambda$ will be called generic.

Remark 3.9 Our terminology generic is justified as follows. If the domain $X$ of $f$ is irreducible over $\mathbb{Q}$, then there is a non-empty Zariski open locus in the Grassmannian variety consisting of generic linear spaces $\Lambda \subset \mathbb{P}^{n}$ of codimension $\delta$, provided $\delta \leq$ $\operatorname{dim} X-\operatorname{dim} \overline{\operatorname{Im}}(f)$. Indeed, by dimension count, $\Lambda$ will intersect all fibers of $f$ and it is enough to assure that it intersects a general fiber not only in the base locus. Thus, if we define $\Lambda$ by picking arbitrary rational coefficients, we will obtain a generic space with probability one-we may only fail if the coefficients satisfy a fixed polynomial relation.

Let $\delta:=\operatorname{dim} X-\operatorname{dim} \operatorname{Im}(f)$ and pick a generic codimension $\delta$ linear section $X^{\prime}:=X \cap \Lambda$ of $X$. Blowing up $X^{\prime}$ along the indeterminacy locus of $f^{\prime}:=\left.f\right|_{X^{\prime}}$ gives a resolution $\tilde{f}^{\prime}: \mathrm{Bl}_{f^{\prime}} X^{\prime} \rightarrow \mathbb{P}^{m}$. Computing the images of the exceptional divisors via $\tilde{f}^{\prime}$ gives a frame of $f^{\prime}$. This in turn is a frame for $f$. All these statements will be proved in Lemma 3.10.

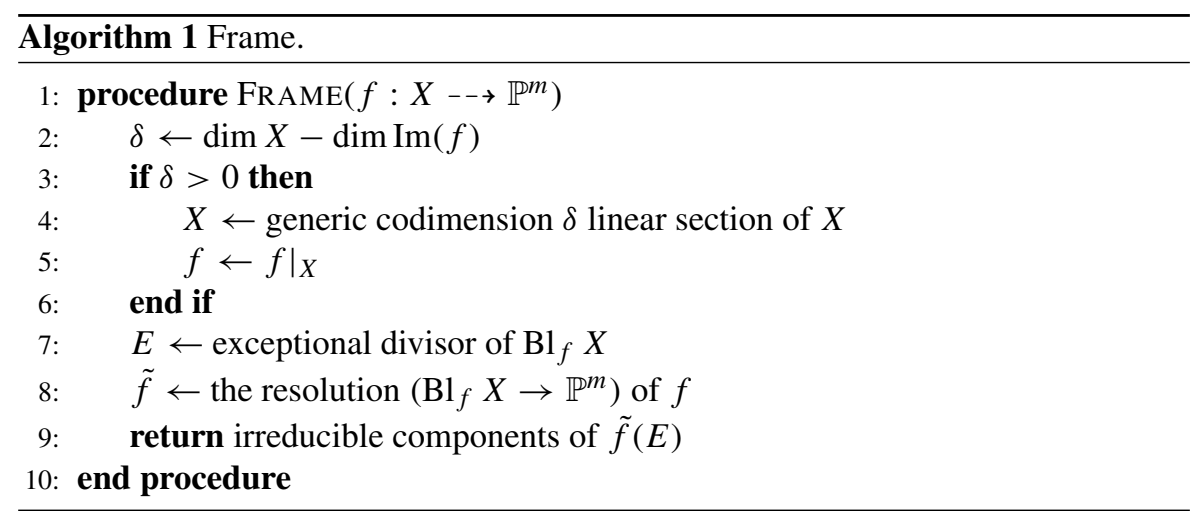

Lemma 3.10 Let $X \subset \mathbb{P}^{n}$ be irreducible. Then Frame $\left(f: X \rightarrow \mathbb{P}^{m}\right)$ returns the irreducible components of a frame A of $f$.

Proof By taking a generic linear section $X^{\prime}$ of $X$, we make sure $\operatorname{dim} X^{\prime}=\operatorname{dim} \operatorname{Im}(f)$ and $f^{\prime}:=\left.f\right|_{X^{\prime}}$ has image closure equal to $\overline{\operatorname{Im}}(f)$. Then the exceptional divisor $E$ of the blowup of $X^{\prime}$ has dimension strictly less than $\overline{\operatorname{Im}}(f)$. Therefore, the image $A$ of $E$ will be strictly contained in $\overline{\operatorname{Im}}(f)$.

On the other hand, $\operatorname{Im}\left(f^{\prime}\right) \subset \operatorname{Im}(f)$, and $\overline{\operatorname{Im}}\left(f^{\prime}\right)=\overline{\operatorname{Im}}(f)$. Therefore, $\overline{\operatorname{Im}}\left(f^{\prime}\right) \backslash$ $\operatorname{Im}\left(f^{\prime}\right) \supset \overline{\operatorname{Im}}(f) \backslash \operatorname{Im}(f)$, and we only need to show the containment $A \supset \overline{\operatorname{Im}}\left(f^{\prime}\right) \backslash$ $\operatorname{Im}\left(f^{\prime}\right)$.

The blowup $\mathrm{Bl}_{f^{\prime}} X^{\prime} \stackrel{\pi}{\rightarrow} X^{\prime}$ gives a resolution $\tilde{f}^{\prime}: \mathrm{Bl}_{f^{\prime}} X^{\prime} \rightarrow \mathbb{P}^{m}$ of $f^{\prime}$. Note that $\operatorname{Im}\left(\tilde{f}^{\prime}\right)=\overline{\operatorname{Im}}\left(f^{\prime}\right)$. In particular, for any point $y \in \overline{\operatorname{Im}}\left(f^{\prime}\right) \backslash \operatorname{Im}\left(f^{\prime}\right)$, we can find a 
point $x \in \tilde{X}^{\prime}$ satisfying $\tilde{f}^{\prime}(x)=y$. Then $\pi(x)$ must be in the indeterminacy locus of $f^{\prime}$. Therefore, $x \in E$ and $y \in A$.

The idea behind the main algorithm Total Image is to compute successively finer approximations of the image boundary $\overline{\operatorname{Im}}(f) \backslash \operatorname{Im}(f)$. We now give an informal demonstration of how these approximations can be obtained.

Let $Y_{0}=\overline{\operatorname{Im}}(f)$ and $A_{0} \supset Y_{0} \backslash \operatorname{Im}(f)$ be a frame of $f$. Then $Y_{0}-A_{0} \subset \operatorname{Im}(f) \subset$ $Y_{0}$. We improve this approximation as follows. Define $X_{1}:=f^{-1}\left(A_{0}\right) \subset X$ to be the preimage of $A_{0}$ and let $Y_{1}=\overline{\operatorname{Im}}\left(\left.f\right|_{X_{1}}\right)$. Note that the image of $\left.f\right|_{X_{1}}$ is precisely $A_{0} \cap \operatorname{Im}(f) \subset Y_{1}$. In particular,

$$
\operatorname{Im}(f)=Y_{0}-A_{0}+\operatorname{Im}(f) \cap A_{0}=Y_{0}-A_{0}+\operatorname{Im}\left(\left.f\right|_{X_{1}}\right) .
$$

Let $A_{1}$ be a frame of $\left.f\right|_{X_{1}}$. This time we have $Y_{1}-A_{1} \subset \operatorname{Im}\left(\left.f\right|_{X_{1}}\right) \subset Y_{1}$. Combining this with Equation (3.11) gives us

$$
Y_{0}-A_{0}+Y_{1}-A_{1} \subset \operatorname{Im}(f) \subset Y_{0}-A_{0}+Y_{1}
$$

The frames are meant to get strictly smaller in dimension. Therefore, after at most $N:=\operatorname{dim} Y_{0}$ iterations, the frame $A_{N}$ should be empty. This gives

$$
Y_{0}-A_{0}+\cdots+Y_{N}-A_{N} \subset \operatorname{Im}(f) \subset Y_{0}-A_{0}+\cdots+Y_{N},
$$

which expresses $\operatorname{Im}(f)$ exactly when $A_{N}=\emptyset$.

Note that our algorithm for computing frames uses the irreducibility of the domain over $\mathbb{Q}$ in a crucial way, since both Remark 3.9 and Lemma 3.10 use this hypothesis. This means that we need to decompose each pullback of a frame into its irreducible components. This causes the algorithm to branch at each step making the construction above harder to visualize. Furthermore, the output will be in the form of a constructible tree. Nevertheless, the nature of the argument remains the same, and we prove in Theorem 3.13 that the resulting constructible tree represents $\operatorname{Im}(f)$.

In preparation for the main algorithm, we introduce the following notions:

1. Assigning varieties to vertices in the algorithm means creating vertices labeled by these varieties.

2. Assigning a set of varieties $\left\{V_{1}, \ldots, V_{k}\right\}$ to children (n) means creating $k$ children of $\mathfrak{n}$ which are labeled with $V_{1}, \ldots, V_{k}$.

3. A vertex is called unprocessed if its label is a subvariety of the domain of $f$.

We are ready to present the main algorithm of this paper, which computes the image of a polynomial map (as we prove in Theorem 3.13). Recall that $\mathcal{B}_{f}$ denotes the indeterminacy locus of $f$. 


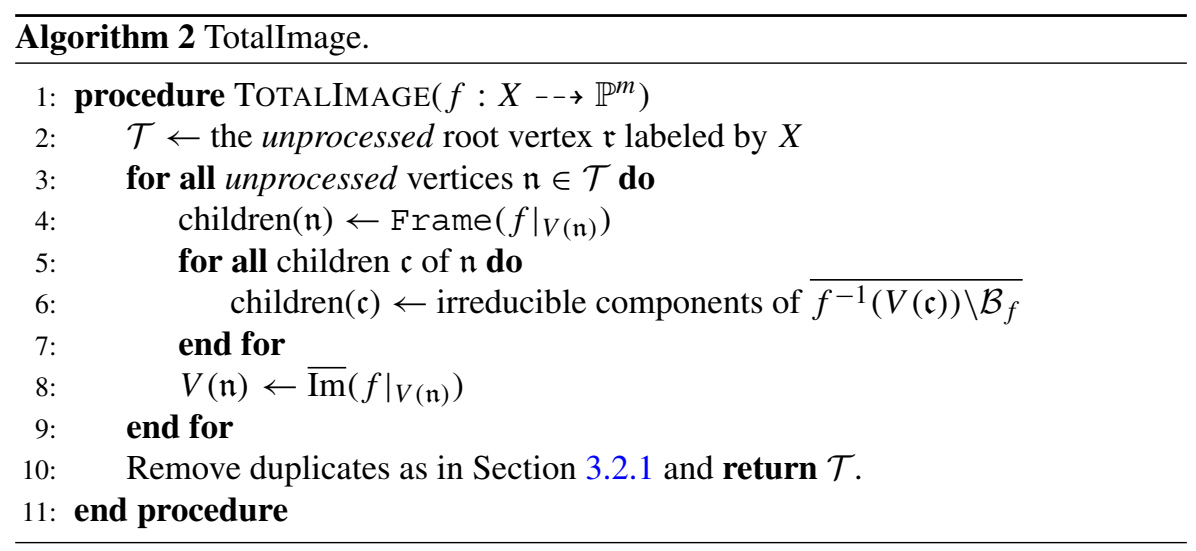

Below, we give a graphical representation of the main loop (line 3) of Total Image. The unprocessed nodes are highlighted $\left(W_{1}, W_{2} \subset X\right)$.

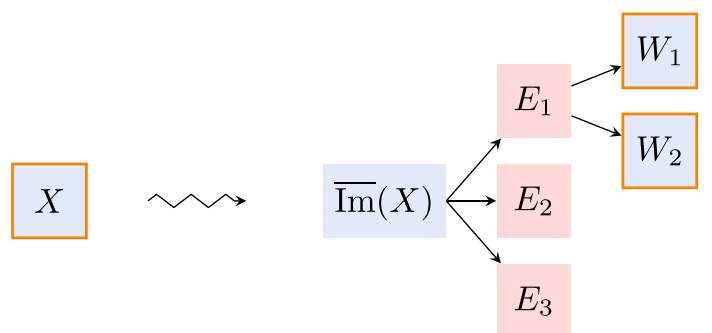

\subsubsection{Cleaning the tree}

The tree we construct at the end of the loop in Total Image may contain edges of the form $\mathfrak{n} \rightarrow \mathfrak{n}^{\prime}$ where $V(\mathfrak{n})=V\left(\mathfrak{n}^{\prime}\right)$. This happens when a component of a frame is dominated by the image. Then we can delete $V(\mathfrak{n})$ as well as all of its descendants and add the descendants of $V\left(\mathfrak{n}^{\prime}\right)$ to the parent of $V(\mathfrak{n})$ (cf. Fig. 4).

There is another instance of redundancy. It may be that $V(\mathfrak{n})$ has two children $V\left(\mathfrak{n}_{1}^{\prime}\right)$ and $V\left(\mathfrak{n}_{2}^{\prime}\right)$ with $V\left(\mathfrak{n}_{1}^{\prime}\right) \subset V\left(\mathfrak{n}_{2}^{\prime}\right)$. It is then unnecessary to keep both of these branches of the tree. We will remove $V\left(\mathfrak{n}_{1}^{\prime}\right)$ and all its descendants (cf. Fig. 5).

\subsubsection{Justification of the algorithm}

Let $f: X \rightarrow \mathbb{P}^{m}$ be a polynomial map. We start by proving a lemma that our algorithm correctly describes the image.
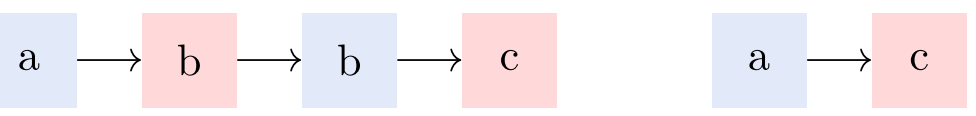

Fig. 4 The tree $\mathcal{T}$ before and after cleaning 

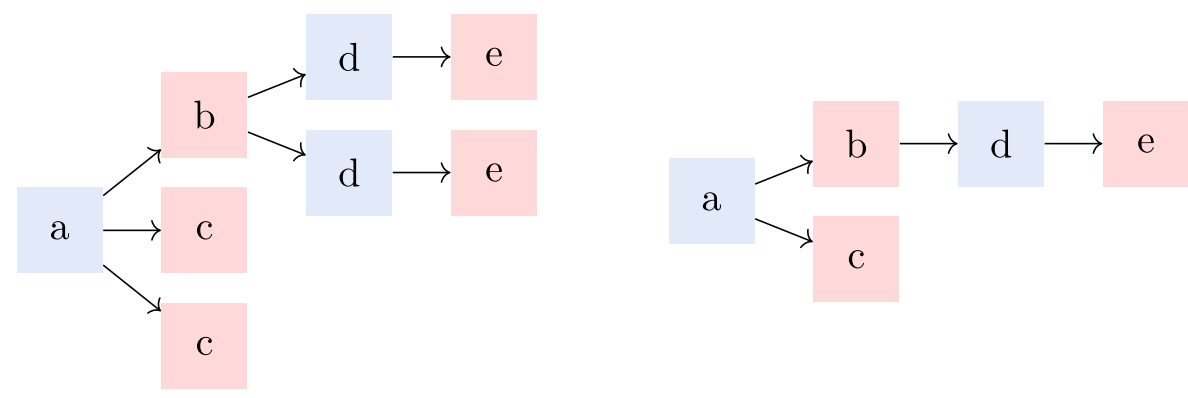

Fig. 5 The tree $\mathcal{T}$ before and after cleaning

Lemma 3.12 Before we clean the tree, for any point $p$ in (resp. not in) the image, any longest path starting at the root and going through vertices labeled by varieties containing $p$ goes through an odd (resp. even) number of vertices.

Proof The proof is inductive on $\operatorname{dim} \operatorname{Im} f$, the case $\operatorname{dim} \operatorname{Im} f=0$ being trivial. If $p \notin$ $\overline{\operatorname{Im}} f$, we are done, so assume $p \in \overline{\operatorname{Im}} f$. If $p$ is not in the image of the exceptional divisor, then $p \in \operatorname{Im} f$ and the claim is true. Otherwise, $p$ belongs to a component $Z$ of the image of the exceptional divisor. Our algorithm will work on components of $f^{-1}(Z)$ and we conclude by induction.

We note that Lemma 3.12 remains true also after cleaning the tree.

Theorem 3.13 The algorithm Total Image terminates and outputs a constructible tree for the canonical representation of $\operatorname{Im}(f)$.

Proof The algorithm stops, as the frames (which always appear at odd levels) have dimension strictly smaller than their parents.

Let $V_{0}-V_{1}+\cdots+(-1)^{\ell} V_{\ell}$ be the canonical representation of $\operatorname{Im}(f)$ as in Definition 2.1. We prove the following statements by induction on $i$ :

1. All components of $V_{i}$ appear at depth $i$ of the tree,

2. All labels at depth $i$ are subvarieties of $V_{i}$.

Note that for $i$ odd (resp. even), the components of $V_{i}$, in the canonical representation, are the largest subvarieties in $V_{i-1}$, with generic points in (resp. not in) $\operatorname{Im} f$. The claim is true for $i=0$. Let $Z$ be a component of $V_{i}$ for $i$ even (resp. odd). Suppose $Z \subset Y$ for $Y$ is a component of $V_{i-1}$. Then $Z$ must be a child of $Y$ by Lemma 3.12, which proves the first point.

For the second point, consider a label $W$ at depth $i$, a child of $Y$. By induction, $Y$ belongs to a component $\tilde{Y}$ of $V_{i-1}$. A generic point of $W$ is (resp. is not) in the image, so $W$ must belong to a component of $V_{i}$.

Corollary 3.14 The algorithm Total Image returns a single node if and only if $\operatorname{Im}(f)=\overline{\operatorname{Im}}(f)$. 


\begin{tabular}{lrrrrrrrrrr}
\hline Example & $\mathrm{I}$ & 2 & 3 & 4 & 5 & 6 & 7 & 8 & 9 & IO \\
\hline Total Image & $\mathrm{O}$ & 0 & 2 & 20 & 0 & 0 & $\mathrm{I}$ & 0 & 29 & 3 \\
PolynomialMapImage & $\mathrm{O}$ & 0 & 22 & $\infty$ & $\infty$ & $\infty$ & $\infty$ & 3237 & $3 \mathrm{I}$ & $\mathrm{I}$ \\
\hline
\end{tabular}

Fig. 6 Timings (in seconds) on an Intel Xeon E7-8837 (2.67 GHz) processor. Runtimes of more than 100 hours are designated by $\infty$

Proof The canonical representation of a set has a single term if and only if the set is closed.

\subsection{Running time}

We compared our implementation with PolynomialMapImage. Now we present timings for comparison (Fig. 6). We used the following examples as benchmarks:

1. The Whitney Umbrella example from documentation ${ }^{2}$ for PolynomialMapImage:

$$
(x, y) \mapsto\left(x y, x, y^{2}\right)
$$

2. The homogenization of the map giving the Whitney Umbrella:

$$
(x, y, z) \mapsto\left(x y, x z, y^{2}\right)
$$

3. The map $G$ given by the gradient of $x y z(x+y+z)$ :

$$
(x, y, z) \mapsto\left(2 x y z+y^{2} z+y z^{2}, x^{2} z+2 x y z+x z^{2}, x^{2} y+x y^{2}+2 x y z\right)
$$

4. The composition $G \circ G$ of the map $G$ from the previous item

5. The map defined by three random ternary cubics

6. The map defined by three random ternary sextics

7. The map defined by three random ternary cubics vanishing on a fixed point

8. The map defined by three random ternary quadrics vanishing on a fixed point

9. The Cavender-Farris-Neyman model (see Section 5.1)

10. The map defining $\operatorname{IMPS}(2,2,3)$ (after restricting the domain) (see Section 5.2)

Let us point out that the two algorithms have outputs of a different nature. As an example, we compare our outputs for item 1 in our list, the Whitney Umbrella. The image is a closed surface in $\mathbb{C}^{3}$. This is demonstrated by the fact that our output has a single node:

(2) ideal $\left(\mathrm{y}^{\wedge} 2 * z-\mathrm{x}^{\wedge} 2\right)$.

However, PolynomialMapImage gives a triangular decomposition, representing the same surface as

$$
\left[\left[x^{\wedge} 2-y^{\wedge} 2 * z\right],[y]\right],[[x, y],[1]] \text {. }
$$

\footnotetext{
${ }^{2}$ https://www.maplesoft.com/support/help/maple/view.aspx?path=RegularChains \%2FConstructibleSetTools\%2FPolynomialMapImage
} 


\section{Site-independent (cyclic) matrix product state}

Matrix product states (MPS) and their more symmetric version-site-independent cyclic matrix product states (IMPS) - play an important role in quantum physics and quantum chemistry [27]. See the Appendix for their definition. These notions are applied, for instance, to compute the eigenstates of the Schrödinger equation. As numerical methods are often involved in their study, the questions of the closedness of families of tensors that allow such representations are central and were asked by W. Hackbusch and L. Grasedyck.

To answer these questions, we present the families of tensors that allow a representation as a matrix product state as orbits under a group action. The equivalence of the classical definition and ours is proven in the Appendix.

We begin by picking a special element in IMPS and describe IMPS as the orbit of this element with respect to change of coordinates. This allows us to work with an explicit parametrization of IMPS, and we will show that this parametrization map does not have a closed image, proving that IMPS is not closed. The element we pick for this purpose is the iterated matrix multiplication tensor.

Since what we do here works equally well over $\mathbb{R}$ or $\mathbb{C}$, we use the letter $\mathbb{K}$ to stand for one of these fields.

Definition 4.1 (Iterated matrix multiplication tensor) For positive integers $a_{1}, \ldots, a_{q}$ define the tensor $\mathfrak{M}_{a_{1}, \ldots, a_{q}} \in \mathbb{K}^{a_{1} \times a_{2}} \otimes \mathbb{K}^{a_{2} \times a_{3}} \otimes \cdots \otimes \mathbb{K}^{a_{q} \times a_{1}}$ as

$$
\mathfrak{M}_{a_{1}, \ldots, a_{q}}:=\sum_{1 \leq i_{j} \leq a_{j}} e_{i_{1}, i_{2}} \otimes e_{i_{2}, i_{3}} \otimes \cdots \otimes e_{i_{q-1}, i_{q}} \otimes e_{i_{q}, i_{1}},
$$

where $e_{i_{j}, i_{j+1}}$ are the basis vectors of the space of matrices $\mathbb{K}^{a_{j} \times a_{j+1}}$.

The following statement may be taken as a working definition of IMPS and MPS. The result itself is a generalization of [18, Proposition 2.0.1].

Proposition 4.2 The sets IMPS and MPS may be represented as

1. $\operatorname{IMPS}(r, k, q)=\left\{f^{\otimes q}\left(\mathfrak{M}_{r, \ldots, r}\right) \mid f \in \operatorname{Hom}\left(\mathbb{K}^{r \times r}, \mathbb{K}^{k}\right)\right\}$,

2. $\operatorname{MPS}(\mathfrak{a}, \mathfrak{b}, q)=\left\{\left(f_{1} \otimes \cdots \otimes f_{q}\right)\left(\mathfrak{M}_{a_{1}, \ldots, a_{q}}\right) \mid f_{i} \in \operatorname{Hom}\left(\mathbb{K}^{a_{i} \times a_{i+1}}, \mathbb{K}^{b_{i}}\right)\right\}$, where $\mathfrak{a}=\left(a_{1}, \ldots, a_{q}\right), \mathfrak{b}=\left(b_{1}, \ldots, b_{q}\right)$.

Proof See Proposition A.5

Remark 4.3 Clearly IMPS $(r, k, q) \subset \operatorname{MPS}((r, \ldots, r),(k, \ldots, k), q)$.

One of the main motivations to start the work on this article was the following question posed by W. Hackbusch:

Question 4.4 Is the set IMPS $(r, k, q)$ closed for every $k, r$, and $q$ ? 
To be more precise, W. Hackbusch expected a negative answer to the above question and also asked for an explicit tensor $T \in \overline{\operatorname{IMPS}(r, k, q)} \backslash \operatorname{IMPS}(r, k, q)$. An analogous question for MPS was asked by L. Grasedyck in the context of quantum information theory and was completely answered in [18].

It is an easy exercise to show that when $q=2$, both IMPS and MPS are closed. Below we will present infinitely many values of $(r, k, q)$ for which $\operatorname{IMPS}(r, k, q)$ is not closed. In fact, we give an explicit tensor $T$ in $\overline{\operatorname{IMPS}(r, k, q)}$ such that $T$ is not even in $\operatorname{MPS}((r, \ldots, r),(k, \ldots, k), q)$, let alone in $\operatorname{IMPS}(r, k, q)$ (see Remark 4.3). This demonstrates that MPS is also not closed in these sets of examples, as predicted by Theorem 1.3.2 of [18].

Using Proposition 4.2, we may describe $\operatorname{IMPS}(r, k, q)$ by the following parametrization map

$$
\psi_{r, k, q}: \operatorname{Hom}\left(\mathbb{K}^{r \times r}, \mathbb{K}^{k}\right) \rightarrow\left(\mathbb{K}^{k}\right)^{\otimes q}: M \mapsto\left(M^{\otimes q}\right)\left(\mathfrak{M}_{r, \ldots, r}\right) .
$$

This puts us exactly within the context of the current article.

We now show that the image of $\psi_{r, k, q}$ is not closed by constructing a point in its closure which is demonstrably not hit by $\psi_{r, k, q}$. Here we will use the idea of approximating the boundary of the image (cf. Section 3.2). In general, the approximation is done by blowing up the indeterminacy locus and computing its image. However, individual points in this approximate boundary may be constructed analytically by approaching the indeterminacy locus along a path

$$
\gamma:(0,1] \rightarrow \operatorname{Hom}\left(\mathbb{K}^{r \times r}, \mathbb{K}^{k}\right)
$$

and computing the $\operatorname{limit}_{\lim _{t \rightarrow 0}} \psi_{r, k, q} \circ \gamma(t)$.

Theorem 4.5 IMPS $(2,4,3)$ is not closed. In fact, there exists a curve $c$ : $(0,1] \rightarrow \operatorname{IMPS}(2,4,3)$ for which $\lim _{t \rightarrow 0} c(t)$ does not even belong to $\operatorname{MPS}((2,2,2),(4,4,4), 3)$.

Proof Let $e_{11}, e_{12}, e_{21}, e_{22}$ be the (standard) basis of $\mathbb{K}^{2 \times 2}$ and $b_{1}, \ldots, b_{4}$ be the basis of $\mathbb{K}^{4}$. We fix an element $M \in \operatorname{Hom}\left(\mathbb{K}^{2 \times 2}, \mathbb{K}^{4}\right)$ which is defined by

$$
M\left(e_{i j}\right)=\left\{\begin{array}{cl}
0 & :(i, j) \neq(1,2) \\
b_{2}: & (i, j)=(1,2) .
\end{array}\right.
$$

Note that $M$ belongs to the indeterminacy locus of $\psi_{2,4,3}$ since $\psi_{2,4,3}(M)=$ $M^{\otimes 3}\left(\mathfrak{M}_{2,2,2}\right)=0$ as can be immediately verified:

$$
M^{\otimes 3}\left(\mathfrak{M}_{2,2,2}\right)=\sum_{i_{1}, i_{2}, i_{3}} M\left(e_{i_{1} i_{2}}\right) \otimes M\left(e_{i_{2} i_{3}}\right) \otimes M\left(e_{i_{3} i_{1}}\right) .
$$

For any term in the summand, the first factor $M\left(e_{i_{1} i_{2}}\right)$ is non-zero if and only if $i_{1}=1$ and $i_{2}=2$. But then the second factor $M\left(e_{i_{2} i_{3}}\right)$ vanishes.

Let $\mathrm{fl} \in \operatorname{Hom}\left(\mathbb{K}^{2 \times 2}, \mathbb{K}^{4}\right)$ be the flattening isomorphism defined by

$$
\mathrm{fl}\left(e_{11}\right)=b_{1}, \operatorname{fl}\left(e_{12}\right)=b_{2}, \operatorname{fl}\left(e_{21}\right)=b_{3}, \operatorname{fl}\left(e_{22}\right)=b_{4} .
$$


Consider the curves $\gamma(t):=(M+t \cdot \mathrm{fl})$ and $c(t):=\frac{1}{t^{2}} \psi_{2,4,3}(\gamma(t))$. Let us denote by $e_{11}^{\vee}, e_{12}^{\vee}, e_{21}^{\vee}, e_{22}^{\vee}$ the dual basis to $e_{11}, e_{12}, e_{21}, e_{22}$. Then we can write

$$
\gamma(t)=t e_{11}^{\vee} \otimes b_{1}+(1+t) e_{12}^{\vee} \otimes b_{2}+t e_{21}^{\vee} \otimes b_{3}+t e_{22}^{\vee} \otimes b_{4} .
$$

From this point onwards, we suppress the tensor notation, writing the tensor product as ordinary product, as no confusion is likely. Recall that we have

$$
\begin{aligned}
\mathfrak{M}_{2,2,2}= & e_{11} e_{11} e_{11}+e_{11} e_{12} e_{21}+e_{12} e_{21} e_{11}+e_{12} e_{22} e_{21} \\
& +e_{21} e_{11} e_{12}+e_{21} e_{12} e_{22}+e_{22} e_{21} e_{12}+e_{22} e_{22} e_{22} .
\end{aligned}
$$

Therefore, we can write $\psi_{2,4,3} \circ \gamma(t)=\gamma(t)^{\otimes 3}\left(\mathfrak{M}_{2,2,2}\right)$ as follows:

$$
\begin{aligned}
\psi_{2,4,3} \circ \gamma(t)= & t^{3} b_{1}^{3}+t^{2}(1+t) b_{1} b_{2} b_{3}+t^{2}(1+t) b_{2} b_{3} b_{1}+t^{2}(1+t) b_{2} b_{4} b_{3} \\
& +t^{2}(1+t) b_{3} b_{1} b_{2}+t^{2}(1+t) b_{3} b_{2} b_{4}+t^{2}(1+t) b_{4} b_{3} b_{2}+t^{3} b_{4}^{3} .
\end{aligned}
$$

It is now clear that $\psi_{2,4,3} \circ \gamma(t) \neq 0$ when $t \neq 0$ so that $\psi_{2,4,3}([\gamma(t)])$ is well defined. We then define:

$$
\begin{aligned}
D & :=\lim _{t \rightarrow 0} c(t) \\
& =b_{1} b_{2} b_{3}+b_{3} b_{1} b_{2}+b_{2} b_{3} b_{1}+b_{4} b_{3} b_{2}+b_{2} b_{4} b_{3}+b_{3} b_{2} b_{4} .
\end{aligned}
$$

We now prove that $D$ is not in MPS. Suppose for contradiction that it were. Then using Proposition 4.2, we can find three linear maps $L_{1}, L_{2}$, and $L_{3}$ in $\operatorname{Hom}\left(\mathbb{K}^{2 \times 2}, \mathbb{K}^{4}\right)$ such that $D=\left(L_{1} \otimes L_{2} \otimes L_{3}\right)\left(\mathfrak{M}_{2,2,2}\right)$. We will now show that each $L_{i}$ is an isomorphism.

Denoting by $V_{i} \subset \mathbb{K}^{4}$ the image of $L_{i}$, we have $D \in V_{1} \otimes V_{2} \otimes V_{3}$ by design. Contracting the second and third tensors via $V_{2}^{\vee}$ and $V_{3}^{\vee}$, respectively, the element $D$ may also be viewed as a linear map

$$
D_{1}: V_{2}^{\vee} \otimes V_{3}^{\vee} \rightarrow V_{1}
$$

However, it is clear that the image of $D_{1}$ is $\left\langle b_{1}, b_{2}, b_{3}, b_{4}\right\rangle$. This forces $V_{1}=\mathbb{K}^{4}$ which in turn implies $L_{1}$ is an isomorphism. Similarly, we can show $L_{2}$ and $L_{3}$ are isomorphisms.

Therefore, $D$ is isomorphic to $\mathfrak{M}_{2,2,2}$. The multiplication tensor $\mathfrak{M}_{2,2,2}$ is known to have tensor rank $7[12,14,15,30]$, but we already have a rank 6 decomposition of $D$, a contradiction.

We stated Theorem 4.5 in a way that the proof could be written explicitly. However, with minor modification, the proof extends to the case of arbitrary odd $q$.

Theorem 4.6 $\operatorname{IMPS}(2,4, q)$ is not closed whenever $q>1$ is odd.

Proof Here we will simply outline the proof in comparison with the proof of Theorem 4.5. Take the same $M \in \operatorname{Hom}\left(\mathbb{K}^{2 \times 2}, \mathbb{K}^{4}\right)$ and $\gamma(t)=M+t \cdot$ fl. Let $\mathfrak{M}_{2, \ldots, 2}$ 
be the iterated matrix product tensor with 2 repeated $q$ times. As before, define the tensor

$$
D:=\lim _{t \rightarrow 0} \frac{1}{t^{\left\lceil\frac{q}{2}\right\rceil}} \circ \gamma(t) .
$$

It will be sufficient to show $D$ is not in MPS. However, the contraction maps $D_{i}$ induced by $D$ all have surjective images when $q$ is odd. Therefore, $D$ is in MPS if and only if $D$ is isomorphic to $\mathfrak{M}_{2, \ldots, 2}$. But $D$ has ranked at most $2 q$ whereas $\mathfrak{M}_{2, \ldots, 2}$ has ranked at least $2^{q-1}[2$, Proposition 20].

\section{Examples}

\subsection{The Cavender-Farris-Neyman model}

The examples below are inspired by statistics. They represent a type of group-based model, which is a special Markov process on trees [20, 26].

The map $\varphi: \mathbb{C}^{6} \rightarrow \mathbb{C}^{8}$ defined below represents the Cavender-Farris-Neyman model (also known as the 2-state Jukes-Cantor model) for the tripod [1]:

$$
\begin{aligned}
(a, b, c, d, e, f) \mapsto \quad & (a c e+b d f, a c f+b d e, a d e+b c f, b c e+a d f, \\
& b d e+a c f, b c f+a d e, a d f+b c e, b d f+a c e) .
\end{aligned}
$$

There are four obvious independent linear phylogenetic invariants-linear polynomials vanishing on the image. In fact, it is well known that the closure of the image is a four-dimensional linear space $[1,26]$. Our algorithm Total Image $(\varphi)$ returns the output in Fig. 7. There are four linear spaces of dimension three, whose generic points do not belong to the image closure $\overline{\operatorname{Im}}(\varphi)$. There are six distinct planes which

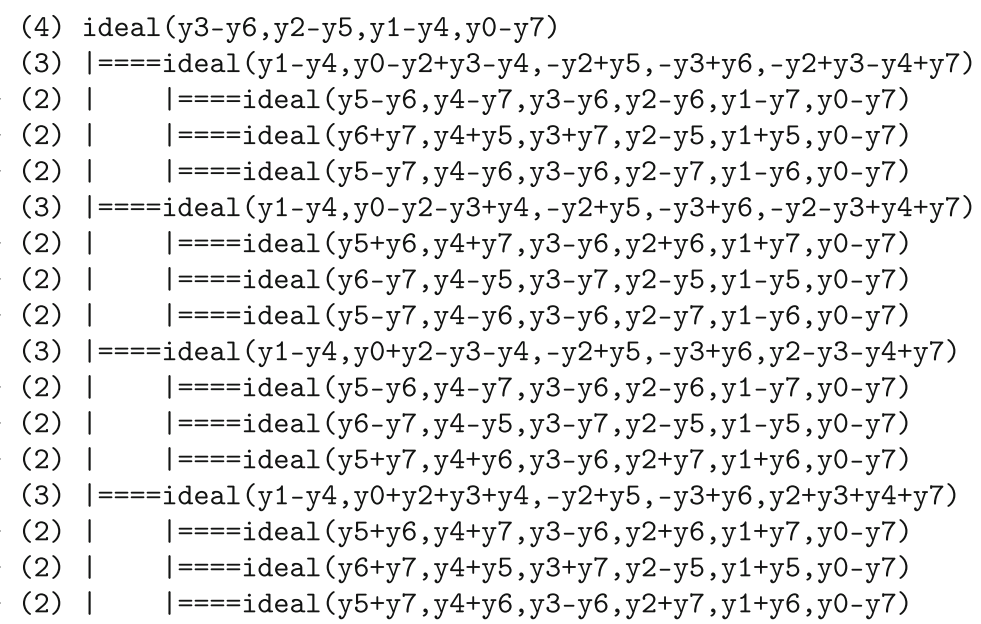

Fig. 7 Total Image output for $\varphi$ 
are added back in. This provides a complete description of the image in statistically meaningful coordinates (without applying the discrete Fourier transform).

As the pairs of parameters $(a, b),(c, d)$, and $(e, f)$ represent probabilities, we may add conditions $a+b=1, c+d=1$, and $e+f=1$. It is known [4] that this adds exactly one additional linear constraint to the closure of the image; namely, that all coordinates sum up to a constant. Further, from this three-dimensional affine space, we have to subtract three two-dimensional subspaces and add to each twodimensional subspace a line and a point.

\subsection{The locus IMPS $(2,2,3)$ is closed}

Here we describe the map $\psi_{2,2,3}$ explicitly in coordinates. An element in the domain of $\psi_{2,2,3}$ is a pair of $2 \times 2$ matrices $(M, L)$ which we write as

$$
\left(\left[\begin{array}{ll}
A & B \\
C & D
\end{array}\right],\left[\begin{array}{ll}
a & b \\
c & d
\end{array}\right]\right) .
$$

The map $\psi_{r, k, q}$ takes this to the coordinate vector

$$
\left[\begin{array}{l}
\operatorname{tr}(M M M) \\
\operatorname{tr}(L M M) \\
\operatorname{tr}(M L M) \\
\operatorname{tr}(M M L) \\
\operatorname{tr}(L L M) \\
\operatorname{tr}(L M L) \\
\operatorname{tr}(M L L) \\
\operatorname{tr}(L L L)
\end{array}\right]=\left[\begin{array}{l}
A^{3}+3 A B C+3 B C D+D^{3} \\
A^{2} a+A B c+a B C+B c D+A b C+B C d+b C D+D^{2} d \\
A^{2} a+A b C+A B c+B C d+a B C+b C D+B c D+D^{2} d \\
A^{2} a+a B C+A b C+b C D+A B c+B c D+B C d+D^{2} d \\
A a^{2}+A b c+a B c+B c d+a b C+b C d+b c D+D d^{2} \\
A a^{2}+a B c+a b C+b c D+A b c+B c d+b C d+D d^{2} \\
A a^{2}+a b C+A b c+b C d+a B c+b c D+c d B+D d^{2} \\
a^{3}+3 a b c+3 b c d+d^{3}
\end{array}\right] .
$$

There are 4 linear relations among these polynomials which implies that the image lies in a four-dimensional subspace $U$ of $\mathbb{C}^{8}$. In fact, $U=S^{3}\left(\mathbb{C}^{2}\right)$ is the subspace of symmetric tensors. We proved that the image is closed and equals $U$ using Total Image in the following way. We restrict the map to pairs of matrices $(M, L)$ where $M$ is diagonal and $L$ has its non-diagonal entries equal. Then Total Image can compute that the image, even restricted to this smaller domain, is exactly $U$. In this example, one could also conclude purely theoretically that the image is closed, as the space of symmetric tensors $U$ has only three $G L(2)$ orbits.

Acknowledgments Open access funding provided by Max Planck Society. We thank Wolfgang Hackbusch for posing the question which motivated this work and for the stimulating discussions. We are grateful to Bernd Sturmfels and Michael Joswig for many suggestions and encouraging remarks.

Funding information MM was supported by Polish National Science Center project 2013/08/A/ST1/00804 affiliated at the University of Warsaw.

Open Access This article is distributed under the terms of the Creative Commons Attribution 4.0 International License (http://creativecommons.org/licenses/by/4.0/), which permits unrestricted use, distribution, and reproduction in any medium, provided you give appropriate credit to the original author(s) and the source, provide a link to the Creative Commons license, and indicate if changes were made. 


\section{Appendix : Matrix product states}

We recall here two representations of tensors that are inspired from physics [23]. The corresponding notions have found applications in deep learning where they are called tensor train or tensor ring decompositions [22, 28, 29, 32].

The symbol $\mathbb{K}$ denotes the field $\mathbb{C}$ or $\mathbb{R}$. For any $a \in \mathbb{Z}_{>0}$, the vector space $\mathbb{K}^{a}$ comes with the standard basis $e_{1}, \ldots, e_{a}$. Therefore, a tensor $T \in \mathbb{K}^{a_{1}} \times \cdots \times \mathbb{K}^{a_{q}}$ may be represented as

$$
T=\sum_{1 \leq i_{j} \leq a_{j}} \lambda_{i_{1}, \ldots, i_{q}} e_{i_{1}} \otimes \cdots \otimes e_{i_{q}},
$$

which is also written

$$
T\left[i_{1}, \ldots, i_{q}\right]=\lambda_{i_{1}, \ldots, i_{q}}
$$

Definition A.1 (Site-independent (cyclic) matrix product state) Fix integers $r>0$, $k>0, q>1$, and matrices $M_{i} \in \mathbb{K}^{r \times r}$ for $i=1, \ldots, k$. Let $T \in\left(\mathbb{K}^{k}\right)^{\otimes q}$ be a tensor given by

$$
T\left[i_{1}, \ldots, i_{q}\right]:=\operatorname{tr}\left(M_{i_{1}} M_{i_{2}} \cdots M_{i_{q}}\right) .
$$

The set of all tensors that allow such a representation will be denoted by $\operatorname{IMPS}(r, k, q) \subset\left(\mathbb{K}^{k}\right)^{\otimes q}$.

Example A.l Let us consider the case of matrices $(q=2)$. Here elements of $\operatorname{IMPS}(r, k, 2)$ can be viewed as matrices $M$ such that $M\left[i_{1}, i_{2}\right]=\operatorname{tr}\left(M_{i_{1}} M_{i_{2}}\right)$. This is equivalent to a factorization of $M=A \cdot A^{t}$ for some matrix $A \in \operatorname{Hom}\left(\mathbb{K}^{r}, \mathbb{K}^{k^{2}}\right)$. In particular, $M \in \operatorname{IMPS}(r, k, 2)$ if and only if $M$ is symmetric and has ranked at most $k^{2}$. It follows that $\operatorname{IMPS}(r, k, 2)$ is closed.

When $q=2$, the tensor $T$ corresponds to a symmetric matrix. However, for $q>2$, the tensor $T$ will not be a symmetric tensor in general, though the identity $T\left[i_{1}, \ldots, i_{q}\right]=T\left[i_{q}, i_{1}, \ldots, i_{q-1}\right]$ continues to hold. In other words, the tensor has cyclic symmetries with respect to the order of the product of the matrices.

Definition A.1 can be regarded as a symmetrization of the following definition of a cyclic matrix product state, where the underlying graph for the tensor network is a cycle.

Fix an integer $q>1$ and tuples of positive integers $\mathfrak{a}=\left(a_{1}, \ldots, a_{q}\right), \mathfrak{b}=$ $\left(b_{1}, \ldots, b_{q}\right)$. We set $a_{q+1}=a_{1}$. Then the locus $\operatorname{MPS}(\mathfrak{a}, \mathfrak{b}, q) \subset \mathbb{K}^{b_{1}} \otimes \cdots \otimes \mathbb{K}^{b_{q}}$ is given by the following definition.

Definition A.3 (Cyclic matrix product state) A tensor $T \in \mathbb{K}^{b_{1}} \otimes \cdots \otimes \mathbb{K}^{b_{q}}$ is in $\operatorname{MPS}(\mathfrak{a}, \mathfrak{b}, q)$ if there exist matrices

$$
M_{i, j} \in \operatorname{Hom}_{\mathbb{K}}\left(\mathbb{K}^{a_{j}}, \mathbb{K}^{a_{j+1}}\right), \quad j=1, \ldots, q, i=1, \ldots, b_{j},
$$

such that

$$
T\left[i_{1}, \ldots, i_{q}\right]:=\operatorname{tr}\left(M_{i_{1}, 1} M_{i_{2}, 2} \cdots M_{i_{q}, q}\right)
$$


Example A.4 The situation for $q=2$ is analogous to Example A.2. In this case, we have $M \in \operatorname{MPS}\left(\left(a_{1}, a_{2}\right),\left(b_{1}, b_{2}\right), 2\right)$ if and only if $M=A B$ where $A \in$ $\operatorname{Hom}\left(\mathbb{K}_{1}^{b}, \mathbb{K}^{a_{1} a_{2}}\right)$ and $B \in \operatorname{Hom}\left(\mathbb{K}^{a_{1} a_{2}}, \mathbb{K}^{b_{2}}\right)$. This can happen if and only if the rank of the matrix $M$ is at most $a_{1} a_{2}$. Therefore, $\operatorname{MPS}(\mathfrak{a}, \mathfrak{b}, 2)$ is always closed.

\section{Proposition A.5 The sets IMPS and MPS may be represented as}

1. $\operatorname{IMPS}(r, k, q)=\left\{f^{\otimes q}\left(\mathfrak{M}_{r, \ldots, r}\right) \mid f \in \operatorname{Hom}\left(\mathbb{K}^{r \times r}, \mathbb{K}^{k}\right)\right\}$.

2. $\operatorname{MPS}(\mathfrak{a}, \mathfrak{b}, q)=\left\{\left(f_{1} \otimes \cdots \otimes f_{q}\right)\left(\mathfrak{M}_{a_{1}, \ldots, a_{q}}\right) \mid f_{i} \in H o m\left(\mathbb{K}^{a_{i} \times a_{i+1}}, \mathbb{K}^{b_{i}}\right)\right\}$.

Proof The proofs of both statements are similar. We prove the first one, as it is more important for this paper. We will be interpreting elements of $\operatorname{Hom}\left(\mathbb{K}^{r \times r}, \mathbb{K}^{k}\right)$ as $r^{2} \times k$ matrices. First, we note that there is a natural bijection $\varphi$ between $k$-tuples of $r \times r$ matrices $\mathcal{M}:=\left(A_{1}, \ldots, A_{k}\right)$ and matrices $\varphi(\mathcal{M}) \in \operatorname{Hom}\left(\mathbb{K}^{r \times r}, \mathbb{K}^{k}\right)$. For $1 \leq i \leq$ $k$, the $i$ th column of $\varphi(\mathcal{M})$ is the representation of $A_{i}$ as a vector of length $r^{2}$.

Write $M_{i}=\sum_{p, q=1}^{r} a_{i, p, q} e_{p, q}$, where $e_{p, q}$ is the matrix with 1 in its $(p, q)$ th entry and zeros everywhere else. Note that $\varphi(\mathcal{M})\left(e_{p, q}\right)=a_{i, p, q}$.

We prove the claim by showing that the tensor $T \in \operatorname{IMPS}(r, k, q)$ associated to $\mathcal{M}$ equals $\varphi(\mathcal{M})\left(\mathfrak{M}_{r, \ldots, r}\right)$. Indeed, we have

$$
\begin{aligned}
T & =\sum_{1 \leq i_{j} \leq k} \operatorname{tr}\left(M_{i_{1}} \cdots M_{i_{q}}\right) e_{i_{1}} \otimes \cdots \otimes e_{i_{q}} \\
& =\sum_{1 \leq i_{j} \leq k}\left(\sum_{1 \leq p_{j} \leq r} a_{i_{1}, p_{1}, p_{2}} a_{i_{2}, p_{2}, p_{3}} \cdots a_{i_{q-1}, p_{q-1}, p_{q}} a_{i_{q}, p_{q}, p_{1}}\right) e_{i_{1}} \otimes \cdots \otimes e_{i_{q}},
\end{aligned}
$$

where in all sums $1 \leq j \leq q$. We can simplify further:

$$
\begin{aligned}
T & =\sum_{\substack{1 \leq i_{j} \leq k \\
1 \leq p_{j} \leq r}}\left(a_{i_{1}, p_{1}, p_{2}} e_{i_{1}}\right) \otimes \cdots \otimes\left(a_{i_{q}, p_{q}, p_{1}} e_{i_{q}}\right) \\
& =\sum_{1 \leq p_{j} \leq r}\left(\sum_{1 \leq i_{1} \leq k} a_{i_{1}, p_{1}, p_{2}} e_{i_{1}}\right) \otimes \cdots \otimes\left(\sum_{1 \leq i_{q} \leq k} a_{i_{q}, p_{q}, p_{1}} e_{i_{q}}\right) \\
& =\sum_{1 \leq p_{j} \leq r} \varphi(\mathcal{M})\left(e_{p_{1}, p_{2}}\right) \otimes \cdots \otimes \varphi(\mathcal{M})\left(e_{p_{q}, p_{1}}\right) \\
& =\varphi(\mathcal{M})^{\otimes q}\left(\mathfrak{M}_{r, \ldots, r}\right) .
\end{aligned}
$$

\section{References}

1. Buczyńska, W., Wiśniewski, J.: On the geometry of binary symmetric models of phylogenetic trees. J. Europ. Math. Soc. 9(3), 609-635 (2007)

2. Buhrman, H., Christandl, M., Zuiddam, J.: Nondeterministic quantum communication complexity: The cyclic equality game and iterated matrix multiplication, arXiv:1603.03757 
3. Chen, C., Lemaire, F., Li, L., Maza, M., Pan, W., Xie, Y.: The ConstructibleSetTools and ParametricSystemTools modules of the RegularChains library in maple. In: International Conference on Computational Sciences and Its Applications, 2008, ICCSA’08, pp. 342-352. IEEE (2008)

4. Chor, B., Hendy, M., Holland, B., Penny, D.: Multiple maxima of likelihood in phylogenetic trees: an analytic approach. Mol. Biol. Evol. 17(10), 1529-1541 (2000)

5. Cox, D., Little, J., O’Shea, D.: Ideals, Varieties, and Algorithms. Springer (1992)

6. Drton, M., Sullivant, S.: Algebraic statistical models. Stat. Sin., 1273-1297 (2007)

7. De Silva, V., Lim, L.: Tensor rank and the ill-posedness of the best low-rank approximation problem. SIAM J. Matrix Anal. Appl. 30(3), 1084-1127 (2008)

8. Grayson, D.R., Stillman, M.E.: Macaulay2, a software system for research in algebraic geometry. Available at http://www.math.uiuc.edu/Macaulay2/

9. Ganter, B.: Algorithmen zur formalen Begriffsanalyse, Beiträge zur Begriffsanalyse (Darmstadt, 1986), pp. 242-254. Bibliographisches Inst., Mannheim (1987)

10. Geiger, D., Meek, C., Sturmfels, B.: On the toric algebra of graphical models. Ann. Stat. 34(3), 14631492 (2006)

11. Hackbusch, W.: Tensor Spaces and Numerical Tensor Calculus. Springer Science \& Business Media (2012)

12. Hauenstein, J., Ikenmeyer, C., Landsberg, J.M.: Equations for lower bounds on border rank. Exp. Math. 22(4), 372-383 (2013)

13. Hampe, S., Joswig, M., Schröter, B.: Algorithms for Tight Spans and Tropical Linear Spaces, arXiv: 1612.03592

14. Hopcroft, J., Kerr, L.: On minimizing the number of multiplications necessary for matrix multiplication. SIAM J. Appl. Math. 20(1), 30-36 (1971)

15. Landsberg, J.M.: The border rank of the multiplication of $2 \times 2$ matrices is seven. J. Am. Math. Soc. 19(2), 447-459 (2006)

16. Landsberg, J.M.: Tensors: Geometry and Applications. American Mathematical Society (2012)

17. Landsberg, J.M., Michałek, M.: On the geometry of border rank decompositions for matrix multiplication and other tensors with symmetry. SIAM J. Appl. Algebra Geom. 1(1), 2-19 (2017)

18. Landsberg, J.M., Qi, Y., Ye, K.: On the geometry of tensor network states. Quant. Inf. Comput. 12(34), 346-354 (2012)

19. Monagan, M., Geddes, K., Heal, K., Labahn, G., Vorkoetter, S., McCarron, J., DeMarco, P.: Maple 10 Programming Guide. Maplesoft, Waterloo (2005)

20. Michałek, M.: Geometry of phylogenetic group-based models. J. Algebra 339(1), 339-356 (2011)

21. Michałek, M.: Toric varieties in phylogenetics. Dissertationes mathematicae 2015(511), 1-86 (2015)

22. Oseledets, I.V.: Tensor-train decomposition. SIAM J. Sci. Comput. 33(5), 2295-2317 (2011)

23. Perez-Garcia, D., Verstraete, F., Wolf, M., Cirac, J.: Matrix product state representations. Quant. Inf. Comput. 7(5), 401-430 (2007)

24. Qi, Y., Michałek, M., Lim, L.-H.: Complex tensors almost always have best lowrank approximations, to appear in Applied and Computational Harmonic Analysis. https://doi.org/10.1016/j.acha.2018.12.003

25. Shafarevich, I.: Basic Algebraic Geometry, 1. Springer (2013)

26. Sturmfels, B., Sullivant, S.: Toric ideals of phylogenetic invariants. J. Comput. Biol. 12(2), 204-228 (2005)

27. Szalay, S., Pfeffer, M., Murg, V., Barcza, G., Verstraete, F., Schneider, R., Legeza, Ö.: Tensor product methods and entanglement optimization for ab initio quantum chemistry. Int. J. Quantum Chem. 115(19), 1342-1391 (2015)

28. Khrulkov, V., Hrinchuk, O., Oseledets, I.: Generalized tensor models for recurrent neural networks, arXiv: 1901.10801

29. Khrulkov, V., Novikov, A., Oseledets, I.: Expressive power of recurrent neural networks, arXiv: 1711.00811

30. Winograd, S.: On multiplication of $2 \times 2$ matrices. Linear Algebra Appl. 4(4), 381-388 (1971)

31. Wu, W.-T.: A zero structure theorem for polynomial equations solving, MM Research Preprints, 1(2) (1987)

32. Zhao, Q., Zhou, G., Xie, S., Zhang, L., Cichocki, A.: Tensor ring decomposition, arXiv:1606.05535

Publisher's note Springer Nature remains neutral with regard to jurisdictional claims in published maps and institutional affiliations. 